\title{
L'évolution de la pollution agricole des eaux souterraines
}

Une approche par couplage de modèles économiques et biophysiques

Assessing the evolution of agricultural groundwater pollution with coupled economic- biophysical models

Nina Graveline, Jean-Daniel Rinaudo, Sebastien Loubier et Volker Segger

\section{(2) OpenEdition}

\section{Journals}

Édition électronique

URL : http://journals.openedition.org/economierurale/2136

DOI : $10.4000 /$ economierurale. 2136

ISSN : 2105-2581

Éditeur

Société Française d'Économie Rurale (SFER)

Édition imprimée

Date de publication : 5 avril 2009

Pagination : 22-39

ISSN : 0013-0559

Référence électronique

Nina Graveline, Jean-Daniel Rinaudo, Sebastien Loubier et Volker Segger, «L'évolution de la pollution agricole des eaux souterraines », Économie rurale [En ligne], 310 | Mars-avril 2009, mis en ligne le 01 mars 2011, consulté le 30 avril 2019. URL : http://journals.openedition.org/economierurale/2136 ; DOI : 10.4000/economierurale.2136 


\section{L'évolution de la pollution agricole des eaux souterraines}

\section{Une approche par couplage de modèles économiques et biophysiques}

Nina GRAVELINE et Jean-Daniel RINAUDO • Bureau de recherches géologiques et minières, Service Eau, Montpellier

Sébastien LOUBIER • Cemagref, UMR G-Eau, Montpellier

Volker SEGGER • LEL Schwabish Gmund, Allemagne

\section{Introduction}

$\mathbf{E}^{\mathrm{n}}$ réponse à l'augmentation régulière des teneurs en nitrates dans les eaux souterraines et à la suite de la promulgation de la directive nitrate $^{1}$ en 1991, les États membres de l'Union européenne ont mis en place d'importants programmes d'actions visant à réduire les pollutions diffuses d'origine agricole. Un rapport de la Commission européenne (European Commission, 2002) montre cependant que ces mesures n'ont pas été suffisantes pour inverser les tendances et passer sous la limite de potabilité $(50 \mathrm{mg} / \mathrm{l})$ dans les zones vulnérables. Ce constat concerne surtout les eaux souterraines dont certaines sont encore caractérisées par une hausse des concentrations en nitrates. En France, cette tendance à la hausse est essentiellement observée en Alsace et dans le quart nord-ouest du territoire (IFEN, 2004).

La mise en œuvre de la directive cadre européenne sur l'eau (DCE) renforce les préoccupations relatives à cette pollution. $\mathrm{La}$ principale innovation apportée par cette directive est d'imposer une obligation de résultat, à savoir l'atteinte du bon état des eaux superficielles et souterraines à l'horizon 2015 (ou au-delà jusqu'à 2027 dans des cas dérogatoires). Concernant les nitrates, le bon état est défini comme le respect de la norme de potabilité c'est-à-dire $50 \mathrm{mg} / \mathrm{l}$. La première étape de la mise en

1. Directive 91/676/EEC. œuvre de cette directive consiste donc à réaliser un exercice de prospective pour évaluer le risque de non-atteinte du bon état à différents horizons temporels. Si un écart à l'objectif de la directive est anticipé, il conviendra alors de mettre en place des mesures correctives. La réalisation de ce diagnostic prospectif est une étape très importante dans le sens où elle détermine l'ampleur du programme d'actions à mettre en œuvre dans le secteur agricole, pour chacune des masses d'eau. Une erreur de diagnostic pourrait conduire à des coûts importants : si l'importance du problème a été sous-estimée, les actions engagées ne seront pas suffisantes pour atteindre l'objectif environnemental, entrainant des dommages pour les usagers de l'eau, et des pénalités pour l'État français. Si, au contraire, l'importance du problème est surestimée, de coûteuses mesures de lutte contre la pollution par les nitrates seront inutilement mises en place. C'est dans ce contexte que s'inscrit le travail de prospective présenté dans cet article.

Plusieurs approches peuvent être mobilisées pour anticiper l'évolution des teneurs en nitrates. La plus simple consiste à extrapoler les tendances passées en matière d'évolution des concentrations en nitrates, l'hypothèse de prolongation des tendances pouvant être acceptable pour les systèmes aquifères à grande inertie (Rinaudo et al., 2005). Mais cette approche ne permet pas de rendre compte des évolutions de teneurs en nitrates qui pourraient résulter de change- 
ments d'assolements et de pratiques des agriculteurs, changements qui eux-mêmes sont induits par l'évolution de la politique agricole et celle des marchés mondiaux, etc. Pour rendre compte de cette interaction entre la dynamique de l'environnement économique et l'évolution de la qualité des eaux souterraines, le recours aux outils de modélisation s'avère très intéressant. Il s'agit de coupler des modèles économiques et biophysiques permettant de simuler les processus suivants : (i) les choix de production et de pratiques par les exploitations agricoles (modèles économiques) ; (ii) le transfert d'azote dans la zone non saturée (modèles sol-plante) ; (iii) les écoulements d'eau et les flux de nitrates dans les eaux souterraines une fois que le polluant y est parvenu.

Cet article présente le résultat d'un tel exercice de couplage de modèle en se concentrant davantage sur le modèle économique développé, réalisé à l'échelle de la plaine du Rhin supérieur. Il s'intéresse à la nappe des alluvions quaternaires du Rhin qui s'étend sur plus de $4200 \mathrm{~km}^{2}$ entre la France (Alsace) et l'Allemagne (pays de Bade). Elle alimente en eau potable près de trois millions de personnes dont $45 \%$ en Alsace, $35 \%$ dans le pays de Bade en Allemagne et $20 \%$ dans la région de Bâle en Suisse et fournit 50 \% des besoins en eau de l'industrie. En 1997, 15 \% des stations de suivi avaient une concentration supérieure à $50 \mathrm{mg} / \mathrm{l}$ et la valeur guide européenne de $25 \mathrm{mg} / \mathrm{l}$ était dépassée dans $36 \%$ des cas (Région Alsace, 2000). Le coût de cette pollution pour les collectivités locales chargées de la production et distribution d'eau potable a été évalué à 26 millions d'euros entre 1988 et 2002 alors que les coûts indirects pour les ménages (achat d'eau en bouteille) et les industriels étaient dix fois supérieurs (Rinaudo et al., op. cit.) La dernière campagne de suivi de la qualité des eaux souterraines (2003) a cependant montré que les teneurs en nitrates ont tendance à se stabiliser, voire baisser localement.

L'article se concentre plus particulièrement sur les modèles économiques d'ex- ploitations agricoles développés pour simuler l'évolution future des systèmes de production et sur le processus de construction de scénarios de changement global. Dans une première section, l'article présente la méthodologie générale développée, les différents modèles utilisés et passe en revue les différents types de modèles économiques pouvant être mobilisés dans ce contexte et le choix de modélisation économique retenue. La deuxième section présente l'analyse des systèmes de production agricole via la typologie d'exploitation et met en évidence les différences entre les parties allemande et française de la zone d'étude. La troisième section se concentre sur la description et la simulation de l'effet de la réforme de la politique agricole commune (PAC) de 2003 sur les choix de culture et l'émission de nitrates, en soulignant également les différences observées en France et en Allemagne. Enfin, nous présentons la démarche prospective mise en œuvre pour construire des scénarios contrastés d'évolution de l'agriculture à moyen terme et présentons les résultats de simulation réalisés avec les modèles couplés.

Bien que les modèles développés puissent aussi permettre de simuler l'impact d'instruments de gestion et de contrôle de la pollution, cet article n'aborde pas cette problématique et se concentre uniquement sur la construction de scénarios prospectifs de l'occupation du sol et des pratiques agricoles. Le lecteur intéressé se reportera à Graveline et Rinaudo (2007) où l'impact des instruments de type taxe ont été simulés avec les mêmes modèles économiques.

\section{Description des modèles utilisés}

L'exercice de modélisation entrepris vise à représenter trois types de processus :

- un processus de décision économique (choix de cultures et de pratiques par les exploitants agricoles) ;

- un ensemble de processus biophysiques déterminant le transfert des nitrates dans 
les sols et la zone non saturée (interaction sol-plante);

- un ensemble de processus physico-chimiques déterminant les flux d'eau et de nitrates dans l'aquifère (transport et atténuation naturelle).

Cette approche interdisciplinaire est comparable à celle présentée par Lacroix et Mollard (2002) et Lacroix et al. (2005) qui poursuivent un objectif toutefois différent à celui de cet article, car ils évaluent l'efficacité de diverses mesures de réduction des teneurs en nitrates des eaux de recharge de nappe. Nous montrerons ultérieurement qu'elle diffère aussi en matière de prise en compte du risque et des incertitudes.

La première étape de notre projet a donc consisté à identifier les outils de modélisation capables à la fois de représenter chacun des processus et d'être couplés pour simuler l'évolution future de la concentration en nitrate de la nappe. Le choix des modèles a été contraint par la possibilité de spatialiser les paramètres d'entrée et les résultats, afin de tenir compte de l'hétérogénéité des systèmes de production agricoles et de l'en- vironnement naturel (sols, climat, conditions oxydo-réductrices de la nappe, etc.). Cette section décrit d'abord succinctement l'architecture globale de la plateforme de modélisation développée, en présentant les modèles biophysiques et hydrogéologiques utilisés. Elle présente ensuite une revue des modèles économiques pouvant être mobilisés. Enfin, elle décrit la méthodologie générale de modélisation économique développée dans ce projet.

\section{Architecture générale de la plateforme de modélisation}

La plateforme de modélisation développée est constituée de cinq modèles (figure 1) qui opèrent à différentes échelles et fonctionnent avec des pas de temps différents (Casper et al., 2005 ; Graveline et al., 2007 ). Les modèles ne sont pas couplés dynamiquement, ils opèrent indépendamment les uns des autres, chaque modèle utilisant comme entrée les résultats du modèle précédent (figure 1). Un premier modèle est utilisé pour interpoler les données climatiques et estimer la recharge de l'aquifère sur

Figure 1. Architecture générale de la plateforme de modélisation

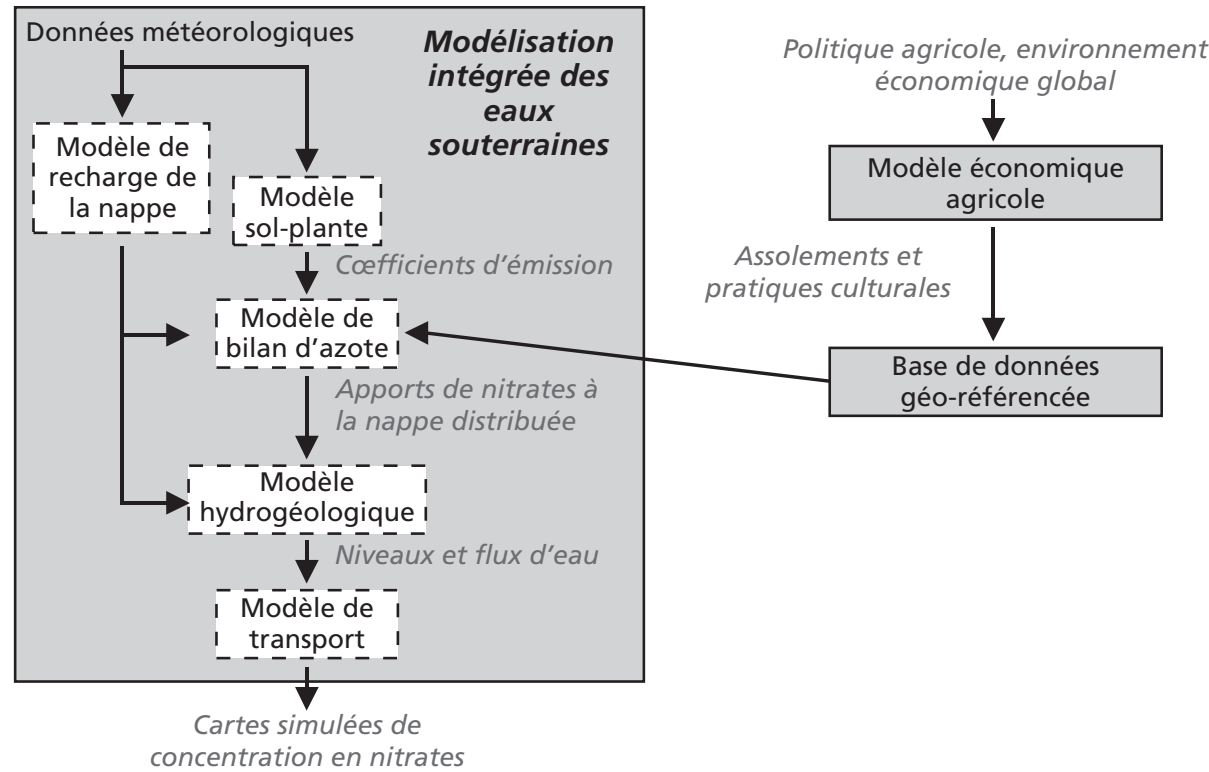

Source : d'après Rinaudo et al. (à paraître)

24 • Économie Rurale 310/Mars-AVRil 2009 
une grille de 500 mètres de coté. En parallèle, le modèle économique (qui sera décrit plus en détail ci-dessous) simule l'évolution des assolements et des pratiques culturales. Les résultats de ces deux premiers modèles alimentent le modèle de bilan d'azote Stoffbilanz qui calcule l'apport en nitrates à la nappe phréatique. Les coefficients de perte par culture et par type de sols sont préalablement calculés en utilisant le modèle biophysique STICS. Les sorties du modèle de bilan d'azote alimentent le modèle hydrogéologique qui simule les flux d'eau et les niveaux piézométriques (avec Modflow) ainsi que le transport et l'atténuation naturelle des nitrates (avec MT3D) dans la nappe.

\section{Choix d'une approche de modélisation économique}

Pour construire des scénarios d'évolution de la production agricole à moyen et long terme, deux types de modèles peuvent être utilisés. Les modèles d'équilibre général (ou partiel) calculables, qui représentent le fonctionnement des marchés, peuvent apporter des éléments de réponse pertinents à une échelle nationale ou supranationale. Le rapport « Prospective 2013 » de l'INRA présente des résultats d'approches de ce type (Guyomard et al., 2007). Les modèles microéconomiques, qui représentent le fonctionnement d'exploitations agricoles, sont plus adaptés pour la construction de scénarios à une échelle locale. Ils présentent en outre l'intérêt de permettre une spatialisation des résultats, et donc une évaluation plus fine de l'impact environnemental des changements de production simulés.

Parmi les méthodes de modélisation micro-économique, nous avons retenu la programmation linéaire, méthode de modélisation normative qui permet de bien représenter les contraintes techniques de production, ce que les modélisations positives ne permettent pas (Ruben et al., 1998). De plus compte tenu de l'échelle relativement fine à laquelle l'analyse des scénarios doit être réalisée, et du fait que les modèles doivent permettre une spatialisation des résultats, seule l'utilisation des modèles de programmation mathématique semblait adaptée. La programmation mathématique linéaire s'avère la plus simple à mettre en œuvre. La rationalité de l'agriculteur y est représentée par la maximisation, sous certaines contraintes d'une fonction d'utilité (i.e. assimilé au profit) ou multicritère (prise en compte éventuel du risque de fluctuation de certains paramètres) (Hazell et Norton, 1986).

Une revue de la littérature récente montre que la programmation linéaire est un outil fréquemment utilisé pour simuler l'évolution probable des choix de production agricoles et leur impact environnemental sur les ressources en eau tant en matière de prélèvement en eau qu'en pollution diffuse. Cette relative popularité des techniques de programmation linéaire pour représenter le comportement des agriculteurs tient en sa simplicité de mise en œuvre et à sa capacité à rendre compte assez finement de la nature des contraintes auxquelles les agriculteurs sont confrontés. Cette finesse est d'autant plus importante que les modèles visent à représenter l'utilisation de ressources naturelles dont la distribution spatiale ou temporelle est hétérogène (l'eau et les types de sols par exemple). C'est également le cas lorsque les modèles visent à représenter finement certains impacts environnementaux de l'agriculture qui ne peuvent être décrits qu'en rendant compte en détail des pratiques agricoles. En outre, cette représentation fine des contraintes et des pratiques permet de mieux interpréter les résultats de simulation à travers notamment l'identification des contraintes actives et les valeurs marginales associées. Cette transparence de la structure et du mode de fonctionnement des modèles permet dans certains cas de les utiliser comme outils d'aide à la négociation (Nidumolu et al., 2007). La diversité des exploitations agricoles est généralement appréhendée à travers la réa- 
lisation d'une typologie des logiques technico-économiques. Un modèle économique est alors développé pour représenter le fonctionnement de chaque type (Rozakis et al., 2001 ; Bartolini et al., 2007). La distribution spatiale des types d'exploitation ou des contraintes liées aux facteurs naturels peut également être prise en compte en couplant les modèles économiques avec un système d'information géographique (Bouman $e t$ al., 1998 ; Zander et Kächele, 1999).

\section{La démarche de modélisation écono- mique retenue}

La première étape de la démarche de modélisation économique a consisté à construire une typologie d'exploitations agricoles visant à rendre compte de la diversité des logiques technico-économiques et du niveau de risque de pollution azotée associé à chaque type d'exploitation. Du côté français, la typologie résulte d'une classification automatique des 14000 exploitations agricoles alsaciennes, basée sur les données du Recensement général agricole de 2000 (Chambre d'agriculture régionale d'Alsace, 2004). Du côté allemand, des experts ont été consultés pour définir des critères de classification hiérarchique (variables et valeurs seuils) et une classification automatique de 9000 exploitations a été réalisée en utilisant les données $\mathrm{PAC}^{2}$ à l'échelle de petites régions agricoles. Le résultat de la classification permet d'évaluer le poids de chaque type d'exploitation en termes de surface agricole utile, d'assolement et de risque de lessivage pour chacune de ces zones.

Pour chacun des principaux types, une exploitation représentative est identifiée avec l'appui des services de l'État et de la profession agricole. Des entretiens détaillés sont ensuite conduits pour décrire finement

2. Le traitement des données a été réalisé par l'organisme instruisant les demandes de subventions (LEL) dans le respect des contraintes de confidentialité des données individuelles. la logique de fonctionnement de cette exploitation. Cette information est utilisée pour développer des modèles micro-économiques de programmation linéaire (PL), qui maximisent la marge brute totale de l'exploitation en prenant en compte un certain nombre de contraintes réglementaires, techniques et de disponibilité de ressources ${ }^{3}$. Ce problème peut être noté mathématiquement sous la forme suivante :

$$
\max \sum_{j=1}^{n} c_{j} X_{j}
$$

en respectant les contraintes :

où

$$
\begin{aligned}
& \sum_{j=1}^{n} a_{i j} X_{j} \leq b_{j} \quad i=1 \ldots n \\
& \text { et } X_{j} \leq 0 \quad j=1 \ldots n
\end{aligned}
$$

$\mathrm{X}_{\mathrm{j}}=$ représente le niveau de l'activité de production $\mathrm{j}$,

$\mathrm{c}_{\mathrm{j}}=$ représente la marge brute de l'activité $\mathrm{j}$,

$\mathrm{a}_{\mathrm{ij}}=$ représente la quantité de ressource $\mathrm{i}$ (par exemple eau, main-d'œuvre, engrais) qui est consommée pour une unité de production $\mathrm{j}$,

$b_{i}=$ la quantité totale de ressource i disponible pour l'exploitation.

Le risque lié à la variabilité interannuelle des rendements et des prix (de 1995 à 2003) dû, respectivement, aux variabilités climatiques et macro-économiques, est aussi pris en compte en utilisant la méthode du MOTAD généralisé (Minimization Of the Total Absolute Deviations) (voir Hazell et Norton 1986 ; Bouzit et al., 1993). À chaque activité, on associe alors la somme des déviations de marge brute (prix et rendement sont les paramètres variables d'une année à l'autre) par rapport à la moyenne sur neuf années (1995-2003). Puis la variation totale est la somme des variations pour chaque production réalisée multipliée par le coefficient de Fischer (qui intègre la

3. Les contraintes sont spécifiées de manière linéaire par rapport aux variables de décisions. 
taille des séries considérées). La variation totale est multipliée par le coefficient d'aversion au risque avant d'être intégrée à la fonction objectif (minimisation du risque lors de l'optimisation). Ce coefficient dépendra du type d'agriculteur et sera obtenu lors du calage des modèles.

La variabilité climatique est prise en compte indirectement au travers des chroniques de rendements de chaque culture de 1995 à 2003. Le risque de lessivage des sols est estimé puis moyenné sur chacune de ces années par le modèle STICS qui prend en compte la variabilité spatiale des sols et leur occupation, mais il n'est pas représenté par une probabilité comme le fait par exemple l'approche développée par Lacroix et al. (2005). On note aussi que le modèle économique ne vise pas, ici, à représenter le choix des agriculteurs en réaction à des instruments de la pollution azotée (voir par exemple Lacroix et al., 2007) seule une fonction de production du maïs et les cultures pièges à nitrate sont intégrées.

La fonction de production du maïs, développée par l'Association pour la Relance agronomique en Alsace sur la base d'essais agronomiques locaux, est aussi intégrée au modèle afin de permettre différents choix sur le niveau de fertilisation azotée et de rendement associé. Les modèles individuels d'exploitations sont calés sur la situation de référence 2003. La validation est réalisée de manière itérative par un groupe d'experts qui analyse la plausibilité des réponses des modèles au vu des résultats de plusieurs simulations.

Les modèles individuels sont ensuite agrégés pour rendre compte des choix de production de l'ensemble du type qu'ils représentent, puis utilisés en simulation pour évaluer l'impact de différents scénarios élaborés par le groupe d'expert. Le résultat des simulations, agrégés à l'échelle de la petite région agricole, servent ensuite d'entrée au modèle de bilan d'azote « Stoffianz ».

\section{Identification des types d'exploitation les plus polluants}

Le premier résultat obtenu est une typologie des systèmes de production. Il s'en suit une estimation du risque de pollution associé à chaque type qui permettra de ne sélectionner pour la modélisation que les types qui contribuent le plus à la pollution.

\section{Résultats de la typologie}

La typologie identifie 16 et 11 types principaux en Alsace et en Bade respectivement (tableau 1). Certains types sont très proches entre les deux pays alors que d'autres sont réellement spécifiques (Graveline et al., 2007). Environ $80 \%$ (Alsace) et $66 \%$ (Bade) des exploitations agricoles qui représentent $85 \%$ et $81 \%$ de la superficie agricole utile totale ont été classés dans l'un des types.

Cinq types céréaliers ont été identifiés $(\mathrm{C} 1$, $\mathrm{C} 2, \mathrm{C} 3, \mathrm{C} 4$ et $\mathrm{C} 5$ ), ils se distinguent entre eux par des tailles et des niveaux de diversification et d'intensification différents. Ils représentent $64 \%$ de la SAU en Bade et $54 \%$ en Alsace.

Les types $\mathrm{C} 1$ et $\mathrm{C} 2$ regroupent des exploitations de plus de 80 ha spécialisées dans la production de maïs (coté français, les exploitations de type $\mathrm{C} 1$ regroupent la quasi-totalité du maïs irrigué). Les exploitations de types C3 sont également des exploitations de grande taille mais plus diversifiées (autres céréales et cultures comme les betteraves ou les oléagineux) et plus présentes en Bade qu'en Alsace. Le type C4 correspond à des exploitations occupant une surface beaucoup plus réduite, (inférieur à 15 ha) mais elles représentent $27 \%$ des exploitations en Bade et $34 \%$ en Alsace. En Bade ces exploitations cultivent davantage de céréales que du maïs, alors que les types alsaciens restent spécialisés en maïs (67\% avec seulement $15 \%$ de céréales). Le type C5 n'est présent qu'en Bade, il est assez diversifié puisqu'il complète ces surfaces en maïs et autres céréales (res- 
pectivement 49 et $15 \%)$ par des cultures spéciales (maraîchage 7 \% ; tabac, vergers, vignes $6 \%$ ) dont il tire le principal de ces revenus. Enfin, de manière générale, l'ensemble de ces types céréaliers cultivent davantage de prairies en Bade qu'en Alsace.

Le type DI est composé d'exploitations diversifiées de taille moyenne (50 ha en moyenne) où la superficie en culture à fortes valeurs ajoutées (betteraves, houblon, tabac) est significative. Le type allemand représente des exploitations bien plus diversifiées (62\% de cultures spéciales en Bade) qu'en Alsace où le maïs demeure la principale culture (67\% contre $30 \%$ en Bade).

La présence plus importante en Bade de cultures spéciales (maraîchage : fraises, asperges ; vergers) demandeuses en travail saisonnier (types C5 en Bade et D1) s'explique par des différences dans la réglementation du travail : l'absence de salaire minimum en Allemagne et une offre de travail agricole en provenance des pays de l'Est de l'Europe (Pologne surtout) «permettent » un prix du travail agricole très peu cher (environ $5 € / \mathrm{h}$ ), rendant les cultures spéciales très rentables.
Les types $M$ (maraîchage) et $H$ (horticulture) sont spécifiques à l'Alsace, mais à l'inverse de leurs voisins badois (D1-M) ils n'occupent que de très petites surfaces (moins de $0,2 \%$ à eux deux).

Les types laitiers ( $\mathrm{L} 1$ et $\mathrm{L} 2$ en Alsace; $\mathrm{L}$ en Bade) ne représentent que $5 \%$ de l'échantillon en Alsace et $1 \%$ en Allemagne mais occupent 15 et $2 \%$ de la surface agricole de chaque région. $\mathrm{L} 1$ est plus intensif que L2 (respectivement 0,7 et 0,4 vaches par ha). La pluspart des surfaces est dédiée à la production de fourrage, les prairies représentent 25 et $40 \%$ respectivement en Alsace et en Bade. Les CIPAN sont mis en place sur $28 \%$ de la surface en Alsace probablement en réponse aux contraintes réglementaires en termes d'épandage.

Les types bovins $\mathrm{B} 1$ et $\mathrm{B} 2$ sont spécialisés dans la production de viande, B1 étant plus intensif que $\mathrm{B} 2$, ces types sont très peu présents (respectivement 6,5 et $4 \%$ de la surface agricole en Bade et Alsace). Les types laitiers et bovins cultivent davantage de prairies en Bade qu'en Alsace, à l'inverse les types alsaciens cultivent plus de

Tableau 1. Importance relative des différents types d'exploitation agricole dans la zone d'étude (tous types)

\begin{tabular}{|c|c|c|c|c|c|c|c|c|c|c|c|c|c|c|c|c|}
\hline \multirow[b]{2}{*}{$\begin{array}{c}\text { Alsace } \\
\text { (France) }\end{array}$} & \multicolumn{4}{|c|}{ Céréaliers } & \multirow[t]{2}{*}{\begin{tabular}{|l|} 
Maraî- \\
chage
\end{tabular}} & \multicolumn{2}{|c|}{ Laitiers } & \multicolumn{2}{|c|}{ Bovins } & \multicolumn{2}{|c|}{ Diversifiés } & \multirow[t]{2}{*}{ Porcs } & \multicolumn{2}{|c|}{$\begin{array}{c}\text { Viti- } \\
\text { culture }\end{array}$} & \multirow[t]{2}{*}{ Ovins } & \multirow[t]{2}{*}{$\begin{array}{l}\text { Horti- } \\
\text { culture }\end{array}$} \\
\hline & $\begin{array}{l}\text { maiss } \\
\text { irrigué }\end{array}$ & $\begin{array}{c}\text { maïs } \\
\text { non } \\
\text { irrigué }\end{array}$ & $\begin{array}{c}\text { poly- } \\
\text { culture }\end{array}$ & $\begin{array}{l}\text { pluri- } \\
\text { actif }\end{array}$ & & $\begin{array}{c}\text { inten- } \\
\text { sif }\end{array}$ & $\begin{array}{c}\text { exten- } \\
\text { sif }\end{array}$ & $\begin{array}{c}\text { inten- } \\
\text { sif }\end{array}$ & $\begin{array}{c}\text { exten- } \\
\text { sif }\end{array}$ & & & & & & & \\
\hline Total $=100 \%$ & C1 & C2 & C3 & C4 & $M$ & L1 & L2 & $B 1$ & $B 2$ & D1 & $D G$ & $D S$ & V1 & V2 & 0 & $H$ \\
\hline $\begin{array}{l}\text { Part du nom- } \\
\text { bre d'exploi- } \\
\text { tation (\%) }\end{array}$ & 5,9 & 3,0 & 0,6 & 34,3 & 0,8 & 2,4 & 3,1 & 0,8 & 2,3 & 5,0 & 0,9 & 0,9 & 26,7 & 11,2 & 0,3 & 2,0 \\
\hline $\begin{array}{l}\text { Part de la } \\
\text { SAU (\%) }\end{array}$ & 20,7 & 9,7 & 2,1 & 21,3 & 0,1 & 5,6 & 9,5 & 2,1 & 4,4 & 11,6 & 1,6 & 2,1 & 2,3 & 6,0 & 0,7 & 0,1 \\
\hline $\begin{array}{c}\text { Pays } \\
\text { de Bade } \\
\text { (Allemagne) }\end{array}$ & Maïsicu & ulture & moyen & olycultt & \begin{tabular}{|} 
ure \\
grand/ \\
cultures \\
spéciales
\end{tabular} & & & $\begin{array}{c}\text { inten- } \\
\text { sif }\end{array}$ & $\begin{array}{c}\text { exten- } \\
\text { sif }\end{array}$ & $\begin{array}{l}\text { Cult } \\
\text { spécia } \\
\text { maraî }\end{array}$ & & Porcs & $\begin{array}{l}\text { Cult } \\
\text { permal } \\
\text { (vero } \\
\text { vign }\end{array}$ & & \begin{tabular}{|c|} 
Petits \\
élevages
\end{tabular} & \\
\hline $\begin{array}{l}\text { Total }=100 \% \\
\text { Part du nom- }\end{array}$ & $\mathrm{C} 1 / \mathrm{C}$ & & C3 & C4 & C5 & L1 & 1 & $B 1$ & $B 2$ & & $-M$ & $D S$ & $v$ & D & $T p$ & \\
\hline $\begin{array}{l}\text { bre d'exploi- } \\
\text { tation (\%) }\end{array}$ & 4,9 & & 4,1 & 26,7 & 10,4 & 0,6 & 6 & 0,5 & 0,4 & & 9 & 0,4 & 14 & 1,5 & 1,9 & \\
\hline $\begin{array}{l}\text { Part de la } \\
\operatorname{SAU}(\%)\end{array}$ & 17, & & 13,8 & 10,7 & 22,5 & 1,8 & 8 & 1,6 & 1,6 & 4 & & 0,7 & 8 & 2 & 0,7 & \\
\hline
\end{tabular}

Source : calculs des auteurs adaptés de la Chambre d'Agriculture région Alsace, 2003 pour la partie Alsace 
maïs. Enfin quelques types spécialisés en productions animales ont été identifiés, il s'agit des types ovins (O) en Alsace, de production de porcs (DS, D2S) et de fermes avicoles (DG).

Les types viticoles $\mathrm{V} 1, \mathrm{~V} 2$ en Alsace et VD (vin et vergers) en Bade sont très nombreux (38\% en Alsace et $14 \%$ en Bade) mais sont, vu leur taille moyenne, moins importants en terme de surface. La différence entre V1 et V2 réside essentiellement dans le niveau de diversification, V1 cultive aussi du maïs et des céréales.

\section{Contribution relative des différents groupes à la nappe}

Nous avons ensuite évalué quelle était la contribution totale de chacun de ces types d'exploitations agricoles en termes d'émission d'azote afin de ne conserver que les types exerçant le plus de pression sur les ressources en eau pour la modélisation. Cette évaluation s'appuie sur l'indicateur suivant :

$$
R_{i}=\sum_{j=1}^{N} \cdot S_{i, j} k_{j}
$$

Où : « $\mathrm{i}$ » indice de type d'exploitation, « $\mathrm{j}$ » indice de type de culture ;

$\mathrm{R}_{\mathrm{i}}$ un indicateur de risque total de fuite azotée pour le type d'exploitation « $\mathrm{i} »$; $\mathrm{S}_{\mathrm{i}, \mathrm{j}}$ la surface en culture « $\mathrm{i} »$ pour le type d'exploitation $« \mathrm{j} »$;

$\mathrm{k}_{\mathrm{j}}$ le reliquat d'azote dans la zone sousracinaire pour la culture $\mathrm{j}$.

Les valeurs de reliquat $\left(\mathrm{k}_{\mathrm{j}}\right)$ sont estimées à partir des 30000 analyses de sol réalisées en Allemagne dans le cadre du programme agro-environnemental «SCHalvo » entre 2001-2003. S'agissant d'analyses réalisées dans des zones de protection des eaux («Wasserschutzgebiet ») et dans un cadre où une présence excessive en nitrates conduit à une pénalité financière de l'agriculteur, les valeurs observées sont supposées faibles par rapport à ce que l'on observerait hors zone de protection.
Les résultats obtenus permettent d'évaluer l'importance relative de la contribution de chaque type d'exploitation à la quantité totale d'azote émise. Ils montrent que six types alsaciens $(\mathrm{C} 1, \mathrm{C} 2, \mathrm{C} 4, \mathrm{D} 1, \mathrm{~V} 2, \mathrm{~L} 1$ et L2) sont à l'origine de plus de $85 \%$ de la pollution émise. Dans la partie allemande de la zone d'étude, six types céréaliers et lait (C1, C2, C3, C4, C5, L) sont à l'origine de $68 \%$ de la pollution émise.

\section{Simulation de l'effet de la réforme de la PAC en France et en Allemagne}

La pression polluante exercée par chaque type d'exploitation sur les ressources en eau souterraine est susceptible d'évoluer à court terme suite à la réforme de la Politique agricole commune. La mise en œuvre de la réforme coïncidant avec la période $\mathrm{du}$ projet, nous avons tenté d'en évaluer l'impact sur les assolements (et par conséquent sur le risque de pollution azotée) à travers l'utilisation des modèles économiques. Les modalités d'application de la réforme de la PAC étant différentes entre la France et 1'Allemagne, des réponses différentes étaient attendues de la part des exploitants agricoles des deux côtés du Rhin. Cette section présente brièvement les modalités de mise en œuvre de la réforme de la PAC en France et au Baden Württemberg avant de présenter quelques résultats de simulation de l'impact de cette réforme.

\section{Des modalités de mise en œuvre contrastées}

Les accords de Luxembourg de 2003 introduisent trois éléments de réforme du mode de soutien de l'agriculture européenne : - le découplage des aides directes par la mise en place du régime de paiement unique aux agriculteurs ;

- l'éco-conditionnalité des aides ;

- la modulation des aides (MAAPAR, 2004 ; Deutscher Bauernverband, 2003). Le principe du découplage consiste à remplacer le système de paiements compensa- 
toires liés à la production par un système de droits au paiement unique indépendant de la production. Le nombre de droits (en ha) est égal à la surface cultivée (condition sur les cultures) pendant la période de référence (2000-2002). La valeur du droit peut être soit uniforme au sein d'une région comme en Allemagne soit basée sur une moyenne historique de l'exploitation (France). Les principales différences entre les modalités de mise en ouvre de cette réforme en France et en Allemagne sont relatives aux trois points suivants :

- Le découplage n'est que partiel en France, $75 \%$ seulement du montant des primes est payé indépendamment de toute production, alors qu'il est total en Allemagne. Ainsi, en France, une incitation à la production de « grandes cultures » est maintenue puisque $25 \%$ du montant des anciennes aides demeure couplé à la production.

- Les différents types de "droit à l'hectare » (montant de prime découplée à l'hectare) : en France on distingue pour un agriculteur des droits « culture » et « jachère »; en Allemagne des droits « prairie » et « culture».
- Le montant de ces droits est aussi calculé de différentes manières en France et en Allemagne. En France le calcul se fait exclusivement sur la base des primes perçues par l'agriculteur pour les années 2000 à 2002. En Allemagne le calcul est plus complexe, il se fait à la fois sur les références individuelles (de l'agriculteur) dans les premières années d'application et sur les références régionales. En 2013, le montant des droits sera régionalisé, c'est-à-dire que tout hectare (ayant droit « prairie » ou « culture ») donnera droit à une prime régionale unique (environ $300 €$ ). Ce système, sera alors relativement incitatif pour les prairies.

\section{Simulation de l'évolution de deux exploitations similaires}

Les modèles économiques développés ont ensuite été utilisés pour simuler l'impact de la réforme de la PAC sur les choix de production, les revenus et le risque global de pollution azotée estimé avec l'indicateur (Indicateur nitrate) décrit ci-dessus. Nous présentons ici les résultats obtenus pour deux exploitations représentatives du type $\mathrm{C} 2$ (grandes exploitations spécialisées en maï-

Tableau 2. Impacts de la réforme de la PAC (2003) sur deux types d'exploitation comparables en Alsace et en Bade

\begin{tabular}{|c|c|c|c|c|}
\hline \multirow[b]{2}{*}{ Productions } & \multicolumn{2}{|c|}{$\mathrm{C} 2 \mathrm{Fr}$} & \multicolumn{2}{|c|}{$\mathrm{C} 2 \mathrm{Bw}$} \\
\hline & Ref. 2003 (ha) & $\begin{array}{l}\text { Réforme } \\
\text { PAC (Var.) }\end{array}$ & Ref. 2003 (ha) & $\begin{array}{l}\text { Réforme } \\
\text { PAC (Var.) }\end{array}$ \\
\hline $\begin{array}{l}\text { SAU } \\
\text { Jachère } \\
\text { Maïs } \\
\text { Blé } \\
\text { Orge } \\
\text { Betteraves } \\
\text { Prairies } \\
\text { CIPAN } \\
\text { Porcs }\end{array}$ & $\begin{array}{r}78,0 \\
7,5 \\
55,0 \\
12,5 \\
0,0 \\
3,0 \\
2,0 \\
12,5\end{array}$ & $\begin{array}{r}0 \% \\
7 \% \\
-11 \% \\
43 \% \\
0 \%\end{array}$ & $\begin{array}{r}100,0 \\
8,7 \\
60,8 \\
16,0 \\
1,5 \\
0,0 \\
13,0 \\
17,5 \\
200\end{array}$ & $\begin{array}{l}0 \% \\
3 \% \\
0 \% \\
0 \% \\
0 \%\end{array}$ \\
\hline \multicolumn{5}{|c|}{ Intrants-facteurs de production } \\
\hline $\begin{array}{l}\text { Main-d'œuvre (h) } \\
\text { Azote minéral (kg) } \\
\text { Gasoil (L) } \\
\text { Indicateur Nitrate }\end{array}$ & $\begin{array}{r}1060 \\
12325 \\
21926 \\
3802 \\
\end{array}$ & $\begin{array}{l}-2 \% \\
-4 \% \\
-5 \% \\
-2 \%\end{array}$ & $\begin{array}{r}1408 \\
15226 \\
18745 \\
4540\end{array}$ & $\begin{array}{l}-0,2 \% \\
-0,4 \% \\
-0,4 \% \\
-0,2 \%\end{array}$ \\
\hline \multicolumn{5}{|l|}{ Résultats économiques } \\
\hline $\begin{array}{l}\text { Marge brute } \\
\text { Valeur de la production }\end{array}$ & $\begin{array}{l}63452 \\
95484\end{array}$ & $\begin{array}{l}-3 \% \\
-4 \%\end{array}$ & $\begin{array}{r}99647 \\
176749\end{array}$ & $\begin{array}{r}-4 \% \\
0 \%\end{array}$ \\
\hline
\end{tabular}


Tableau 3. Impacts de la réforme de la PAC (2003) sur l'ensemble des types modélisés

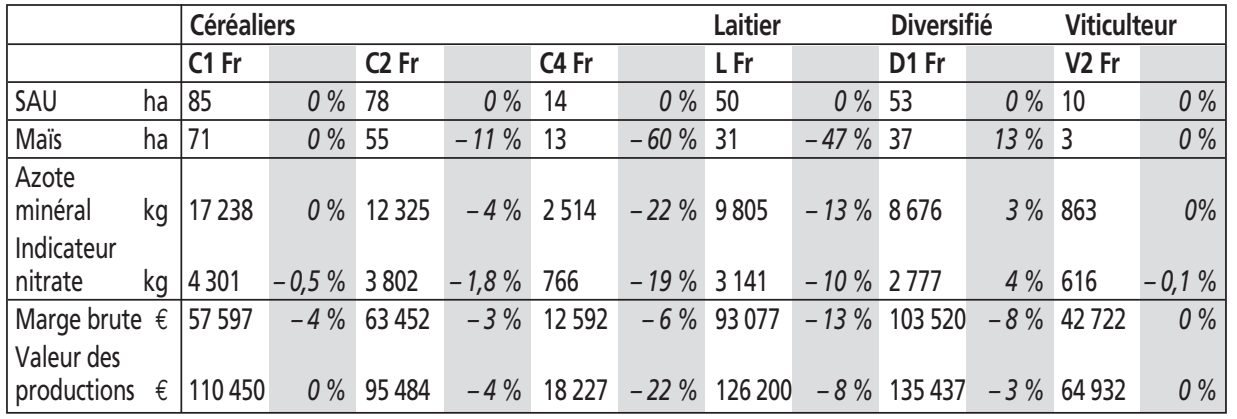

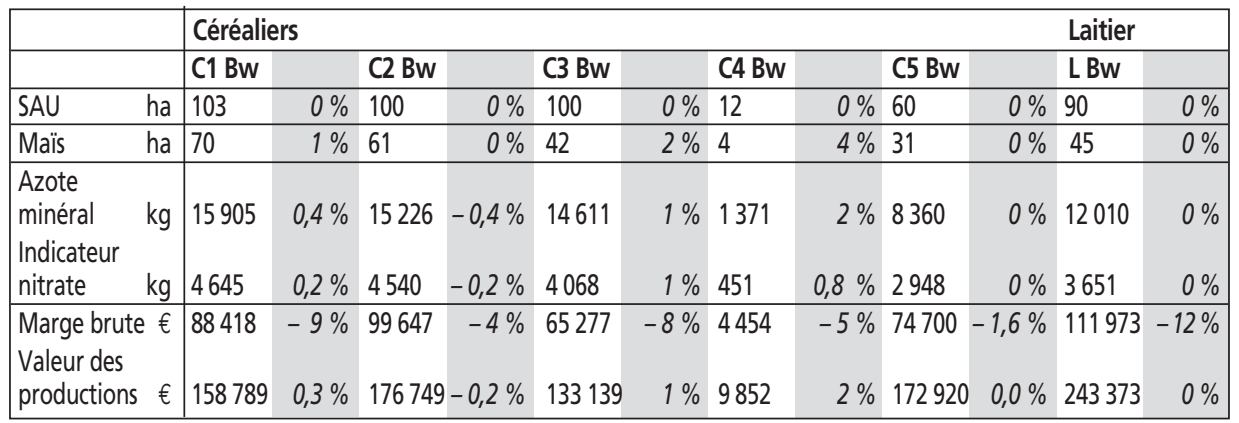

Source : calculs des auteurs

siculture, l'exploitation allemande ayant un petit atelier d'élevage en diversification).

Les résultats de simulation (tableau 2) montrent que la réforme n'a quasiment pas d'impact sur l'assolement pour le type allemand $\mathrm{C} 2$, la valeur relative des marges brutes des cultures restant approximativement inchangées en Allemagne. Les choix de production de l'exploitation alsacienne sont en revanche partiellement modifiés, la surface en blé augmentant au détriment de celle du maïs. Ceci entraine une réduction de l'utilisation d'azote minéral $(-4 \%)$ et permet une augmentation des surfaces en Culture intermédiaire piège à nitrate (CIPAN) réduisant ainsi le risque de pollution azotée ( $-2 \%$ d'émission).

L'observation de l'impact simulé sur l'ensemble des types (voir tableau 3) montre des réactions assez différentes suivant les types. Les assolements sont très fortement modifiés pour les types $\mathrm{C} 4 \mathrm{Fr}$ (petites exploitations grandes cultures, France) et LFr (laitiers, France) qui abandonnent respectivement
$60 \%$ et $47 \%$ des surfaces en maïs au profit du blé. L'impact de la réforme sur le revenu est variable selon les types d'exploitation, variant de 0 à $13 \%$ de réduction de la marge brute totale. Les principaux perdants sont les exploitations laitières (réduction de la marge brute totale de respectivement $13 \%$ et $12 \%$ en France et Allemagne) et les exploitations diversifiées en France $(-8 \%)$. Les résultats de simulations montrent aussi que la réforme de la PAC contribue à réduire les émissions d'azote. L'indicateur de pression (ou « indicateur nitrate » dans le tableau 3) baisse de manière importante pour les petites exploitations françaises en grandes cultures C4 $(-19 \%)$ et pour le type laitier $(-10 \%)$. L'indicateur de pression reste inchangé en Allemagne, reflétant la stabilité des assolements. Enfin, la valeur totale de la production commercialisée baisse de manière significative pour deux types en France (- $22 \%$ pour C4-Fr et $-8 \%$ pour L-Fr) alors qu'elle reste constante en Allemagne. 


\section{Évolution de la qualité de l'eau souterraine pour différents scénarios de changement global}

Les premiers résultats de simulation suggérant que la réforme de la PAC est peu susceptible de modifier le niveau de pression exercée par l'agriculture sur les eaux souterraines, nous avons élargi la réflexion prospective et tenté d'appréhender de manière plus globale les changements susceptibles d'accentuer ou de réduire le risque de pollution à moyen terme. La démarche prospective mise en place a été inspirée par l'approche développée par Alcamo et al. (1996). La première étape a consisté à identifier les facteurs susceptibles de faire évoluer les choix de production et les pratiques des exploitations agricoles, en s'appuyant sur un groupe d'experts agricoles. La seconde étape a consisté à construire trois scénarios contrastés intégrant de manière cohérente les hypothèses d'évolution formulées pour chaque facteur de changement. Enfin, la troisième étape a consisté à simuler l'impact économique et environnemental de ces scénarios, en utilisant les modèles couplés.

\section{Les facteurs de changement}

Vingt-six facteurs de changement ont ainsi été identifiés par le groupe d'experts francoallemand. Ils ont ensuite été hiérarchisés en tenant compte de l'intensité de leur impact sur l'agriculture et de l'incertitude associée à leur évolution. Ce travail de hiérarchisation a permis de sélectionner cinq facteurs principaux (en complément de la réforme de la PAC, telle que décrite ci-dessus) :

- Le risque de prolifération de la chrysomèle du maïs : en 2003, une attaque de ce parasite du maïs a provoqué d'importants ravages sur les cultures de maïs dans la Hardt et le Sundgau (sud de l'Alsace). Ceci a conduit, en 2004, à une réduction de $70 \%$ des surfaces en maïs dans une zone de sécurité de 10000 hectares (mesures réglementaires et de précaution prise par les agricul- teurs). L'ampleur des dégâts occasionnés en région parisienne, dans l'Est de l'Europe ou aux États-Unis conduit à envisager la possibilité d'une prolifération de ce parasite dans le Rhin supérieur et sa prévention conduirait au recul de la monoculture du maïs (nouvelles rotations obligatoires).

- Le prix de l'énergie : la tendance à la hausse du prix des carburants fossiles utilisés en agriculture pourrait entraîner une baisse notable des marges brutes des cultures nécessitant le plus d'opérations mécaniques, d'irrigation ou de séchage, fortement consommatrices en énergie. Le prix des engrais qui dépend fortement de celui de l'énergie pourrait également augmenter (le gaz est la matière première et la ressource énergétique dans la production d'engrais). Cette hypothèse d'évolution du marché de l'énergie pourrait conduire à réduire les objectifs de rendements et d'apports d'azote des agriculteurs et, par voie de conséquence, réduire les émissions de nitrates.

- Le développement des cultures énergétiques: Le contexte énergétique décrit ci-dessus pourrait engendrer un développement de la demande en bioénergies. Deux filières peuvent émerger : la première, en Bade uniquement, consiste à produire du biogaz (usine de transformation) à partir de maïs ; la seconde, consiste à produire des biocarburants (huile végétale pure à partir de colza par extraction sur l'exploitation ou à l'échelle industrielle). À l'échelle de l'exploitation les agriculteurs peuvent ainsi produire leur propre carburant et éventuellement valoriser les tourteaux (coproduits) en alimentation animale. Le développement de ces cultures dépendra largement des politiques économiques (fiscalité et subventions) et des organisations des filières locales, les biocarburants restant à ce jour plus coûteux à produire que le pétrole.

- Les prix et redevance pour l'eau d'irrigation: en Alsace, les superficies irriguées sont très importantes, notamment du fait d'un contexte plus favorable qu'au Baden- 
Württemberg (législation, exonération de la redevance prélèvement en Alsace jusque-là et «Wasserpfennig » en Bade). Or, cette redevance est rétablie (loi sur l'Eau) en application de la directive cadre sur l'eau qui stipule que les états membres doivent mettre en place des tarifications incitatives. Ceci induirait une baisse de rentabilité moyenne du maïs irrigué et des cultures maraîchères pouvant favoriser le développement de cultures alternatives.

- L'élargissement européen risque d'impliquer une augmentation du coût de la maind'œuvre saisonnière en Allemagne. En 2003 il était très bas en raison de la possibilité d'employer des travailleurs agricoles des pays de l'Est (Pologne notamment) sans salaire minimum. L'adhésion à l'Union européenne des pays de l'est européen conduira vraisemblablement, d'ici 2015, à une augmentation progressive du niveau de vie et des salaires dans ces pays, qui se répercutera sur le marché du travail saisonnier en Allemagne. Les marges brutes des cultures maraîchères et fruitières en seront réduites, conduisant, peut-être, à une réduction des surfaces de ces cultures par ailleurs fortement polluantes.

\section{Développement de trois scénarios}

Trois scénarios cohérents, combinant différentes hypothèses d'évolution des facteurs de changement décrits ci-dessus, ont ensuite été élaborés. Un scénario tendanciel, représentant la description la plus plausible de l'évolution de l'environnement économique, réglementaire et naturel a d'abord été élaboré. Puis deux scénarios contrastés ont été développés, en s'inspirant de deux des scénarios (A1 et B2) élaborés par le panel international sur le changement climatique (IPCC, 2000) dans le cadre du rapport spécial sur les scénarios d'émission « Special Report on Emission Scenarios » (SRES).

- Le scénario tendanciel suppose que la réforme de la PAC de 2003 est complètement appliquée à partir de 2013, comme elle est prévue aujourd'hui. La prolifération de la chrysomèle du maïs s'étend à l'ensemble de la zone étudiée, forçant les agriculteurs à adapter leurs rotations culturales (50\% maximum de la SAU est occupée par du maïs). Le prix de l'énergie (gasoil) augmente de $6 \%$ par an en moyenne, atteignant en 2015 le double de sa valeur de 2003 (il est supposé qu'aucun mécanisme de compensation n'est mis en place par l'état). Conséquence directe de la hausse du prix de l'énergie, le prix des engrais azotés augmente de 1,5\% par an. Les prix des phytosanitaires, qui sont très peu dépendant des prix de l'énergie, sont maintenus constants. Le coût de la maind'œuvre agricole saisonnière augmentera suite à l'élargissement européen (soit + 66 \% en douze ans) et affectera les cultures faisant intervenir un travail saisonnier important (arbres fruitiers, légumes, fruits rouges et vigne). En France on admet que les agriculteurs irrigants devront acquitter une redevance d'un montant de $0,025 € / \mathrm{m}^{3}$. En Allemagne, le «Wasserpfennig » est maintenu $\left(0,05 € / \mathrm{m}^{3}\right.$ en 2003). Le développement des biocarburants permettra aux agriculteurs français et allemands de se lancer dans la production d'huile végétale pure sur leur exploitation (incitation du Conseil régional en Alsace). En Allemagne les agriculteurs peuvent aussi vendre leur maïs à l'industrie pour la production de biogaz.

- Le scénario «AI » représente une vision du futur à orientation libérale, il est inspiré du scénario A1 du SRES. Dans cette vision du futur, l'agriculture a pour principal objectif un maintien de sa compétitivité sur des marchés agricoles qui continuent de s'ouvrir à l'international, sans freins protectionnistes et avec un niveau de contraintes environnementales bas. L'augmentation du prix du pétrole en France et en Allemagne évolue à la hausse (40\% en Allemagne et $68 \%$ en France) mais est limitée par un mécanisme de stabilisation des prélèvements fiscaux (taxe flottante française type Taxe intérieure sur les produits 
pétroliers). Le prix des engrais azoté subit la même augmentation que dans le scénario tendanciel. Des moyens techniques importants (traitement par hélicoptère) sont mobilisés par les pouvoirs publics pour éradiquer la chrysomèle et permettent de ne pas limiter les surfaces occupées par du maïs. La logique de libéralisation conduit la France à opter pour un découplage total (au lieu du découplage partiel actuel) plus facile à mettre en œuvre pour les organismes payeurs et gestionnaires. La redevance sur les prélèvements en eau est supprimée en Allemagne et elle n'est pas établie en France. La filière biocarburants se développe. Enfin, le prix de la main-d'œuvre saisonnière augmente, puisque les pays de l'Europe de l'Est ont rejoint l'Union européenne et les salaires agricoles ont augmenté.

- Le dernier scénario «B2 » correspond à une vision du futur dans lequel l'agriculture évolue sous la contrainte de prix des intrants toujours plus élevés et de politiques de protection de l'environnement volontaristes (taxe sur les engrais, augmentation de la redevance eau et pas d'aide ni d'exonération sur la fiscalité de l'énergie). Il est inspiré du scénario B2 du SRES. Le prix de l'énergie augmente de près de $10 \%$ par an en moyenne (pas de mécanisme de stabilisation fiscale) ce qui conduit à tripler le prix en 2015. L'agriculture peut réorienter sa production vers la production de biogaz (Allemagne) ou huiles brutes végétales, rendues plus intéressantes dans ce contexte énergétique. La présence de chrysomèle du maïs oblige les agriculteurs à s'orienter vers des systèmes où le maïs ne revient qu'une année sur trois. La France adopte également le découplage total des aides européenne (PAC). Une politique environnementale volontariste est mise en œuvre à travers la mise en place d'une taxe sur les engrais azotés $(0,15 € / \mathrm{kg}$ d'azote en France et $0,26 € / \mathrm{kg}$ en Allemagne) et la mise en place ou l'augmentation d'une redevance sur les prélèvements en eau $\left(0,025 € / \mathrm{m}^{3}\right.$ au-delà de $7000 \mathrm{~m}^{3} /$ an par forage en France et 0,05
$€ / \mathrm{m}^{3}$ en Allemagne). Enfin, des aides à la mécanisation sont mises en place (investissement) dans les secteurs maraîchers pour soutenir ce secteur menacé par la hausse du coût de la main-d'œuvre temporaire (élargissement européen).

\section{Impacts des scénarios sur les choix de production agricole}

Les conséquences des hypothèses formulées dans les trois scénarios décrits ci-dessus ont été simulées en utilisant les modèles économiques pour les 12 principaux types d'exploitation (tableau 4).

Le scénario tendanciel, décrit comme le plus probable par le groupe d'experts, conduit aux changements suivants par rapport à la situation de référence (2003). Les agriculteurs diversifient leur assolement, remplaçant $26 \%$ des cultures pratiquées en 2003 par d'autres cultures. Les surfaces en maïs reculent largement $(-24 \%$ de la SAU totale) au profit des céréales (essentiellement blé, marginalement orge et céréale d'été) et, dans une moindre mesure au profit du colza qui est transformé en huile végétale pour une autoconsommation en tant que carburant agricole. En Alsace, la marge brute totale dégagée par le secteur agricole modélisé baisse de $11 \%$. Les différents types d'exploitations agricoles ne sont cependant pas impactés de la même manière, puisque le revenu est directement dépendant de leurs productions et de leurs productions passées (pour les aides PAC découplées). C'est le maïsiculteur irrigant qui voit sa marge brute reculer le plus (19\%). Dans le pays de Bade, la perte de marge brute totale est plus importante : elle atteint $27 \%$. La valeur des productions commercialisées baisse peu en Alsace (-3\%) mais davantage en Bade (21\%) en raison de l'abandon relativement important de cultures maraîchères.

Les deux scénarios A1 et B2 s'écartent du scénario tendanciel de manière significative : - le scénario A1 conduit à une moindre modification des assolements que dans le 
Tableau 4. Assolements simulés avec les modèles économiques pour les 12 principaux types d'exploitation agrégés à l'échelle de la zone d'étude

\begin{tabular}{|l|c|c|c|c|}
\hline & $\begin{array}{c}\text { Situation de } \\
\text { référence }\end{array}$ & $\begin{array}{c}\text { Scénario } \\
\text { tendanciel }\end{array}$ & Variante A1 & Variante B2 \\
\hline SAU & $100 \%$ & $100 \%$ & $100 \%$ & $100 \%$ \\
\hline Maïs grain & $58 \%$ & $34 \%$ & $43 \%$ & $19 \%$ \\
\hline Blé & $16 \%$ & $36 \%$ & $30 \%$ & $35 \%$ \\
\hline Colza & $1 \%$ & $6 \%$ & $2 \%$ & $20 \%$ \\
\hline $\begin{array}{l}\text { Cultures industrielles ou assimilées } \\
\text { (betteraves, tabac, légumineuses, } \\
\text { pommes de terre) et maraîchage }\end{array}$ & $6 \%$ & $6 \%$ & $6 \%$ & $6 \%$ \\
\hline $\begin{array}{l}\text { Autres céréales (orge, seigle, blé } \\
\text { d'été) et maïs ensilage }\end{array}$ & $9 \%$ & $10 \%$ & $9 \%$ & $10 \%$ \\
\hline Jachère & $9 \%$ & $9 \%$ & $10 \%$ & $9 \%$ \\
\hline
\end{tabular}

Source : d'après Graveline et al., 2007

scénario tendanciel (17\% de la SAU soit $9 \%$ de moins que dans le scénario tendanciel) et s'accompagne aussi d'un moindre impact sur les marges brutes. La réduction des superficies en maïs reste cependant importante en Alsace (-24\%) et se fait au profit du blé. Les prix du gasoil ne sont pas assez incitatifs pour la production de colza à grande échelle. En Bade, ce sont au contraire les surfaces en blé qui diminuent (ceci concerne $8 \%$ de la SAU et $54 \%$ des surfaces en blé) au profit du maïs qui augmentent de $6 \%$ (soit $+11 \%$ du maïs) par rapport à la situation de référence ;

- le scénario B2 conduit à un changement d'assolement plus important que le scénario de référence ( $42 \%$ des surfaces subissent des changements de cultures, soit $16 \%$ de plus que dans le scénario tendanciel), la monoculture du maiis étant quasiment abandonnée au profit de rotations biennales et triennales (maïs/blé/colza qui se généralisent dans le scénario B2). Ceci est dû à la prolifération de la chrysomèle du maïs, à l'augmentation du prix du gasoil et dans une moindre mesure du prix de l'engrais. Le large développement du colza (pour huile végétale pure) dans le scénario B2 (environnemental) répond directement à la hausse des prix de l'énergie qui incite les agriculteurs à produire du biodiesel pour leur propre consommation. À noter que ceci conduit à une baisse d'environ $8 \%$ des émissions de $\mathrm{CO}_{2}$ liées aux travaux de mécanisation des exploitations concernées (estimation réalisée avec les coefficients cités par Marleix, 2004). Le modèle rend également compte d'une baisse de la fertilisation minérale, les agriculteurs préférant viser un rendement objectif plus faible en réponse à la hausse du coût des engrais.

Avec le scénario tendanciel, les modifications d'assolement sont plus marquées en Alsace : elles concernent $32 \%$ des surfaces cultivées contre $11 \%$ en Bade. Ceci s'explique par le fait que les exploitations badoises sont déjà plus diversifiées que les alsaciennes où le maïs est encore très largement dominant en 2003, et c'est le maïs qui subit la plus forte modification de marge brute. Les types d'exploitation sont aussi plus ou moins sensibles (les viticulteurs par exemple n'évoluent pas).

Le modèle permet également de simuler l'évolution de certaines pratiques agricoles : - Les volumes prélevés en eau (pour le maïs, les cultures maraîchères et le tabac) sont réduits de $50 \%$ dans le scénario A1 et de $80 \%$ dans le scénario B2 en raison de la baisse des surfaces irriguées par le maïs et des baisses des surfaces en légumes (principale consommatrice d'eau agricole en Bade). La réduction des volumes d'eau d'irrigation est plus mar- 
quée en Alsace: le maïs irrigué baisse (A1, tendanciel) ou disparaît (B2).

- Un développement des Cultures intermédiaires piège à nitrate (CIPAN) est également simulé, cette évolution accompagnant la baisse des surfaces en maïs (dont la récolte est généralement trop tardive pour permettre l'implantation d'un couvert herbacé). Avec le scénario tendanciel, les surfaces en CIPAN passent de 12 à $40 \%$ en Alsace et de 20 à $26 \%$ seulement en Bade où la superficie en maïs était initialement plus faible (en pourcentage).

Ces changements importants de production supposent que les filières aval de l'agriculture (agroalimentaire, négoce) absorbent ou se restructurent pour écouler la production de céréales (blé et orge), pour faire face à la baisse de production locale de maïs et trouver des marchés pour les coproduits du colza (tourteau pour l'alimentation animale). En effet les modèles utilisés modélisent l'offre agricole mais ne tiennent pas compte de l'évolution de la demande (puisque les prix sont maintenus constants).

\section{Impacts à long terme sur la concentration en nitrates}

Les conséquences des trois scénarios globaux ont été établies grâce à la chaine des modèles décrite précédemment. Les résultats de ces simulations à l'horizon 2050 ( graphique 1) montrent globalement que tous les scénarios conduisent à une décroissance des teneurs en nitrates dans la nappe et que ces résultats sont peu différents d'un scénario à l'autre à court terme (estimé à 19 mg/l pour le tendanciel et le scenario A1 et 19,5 pour le scenario B2) ce qui peut s'expliquer par le temps de réponse long de l'aquifère aux pressions de surface. Ces différences vont s'accentuer dans le futur. Le modèle montre aussi que les surfaces où la concentration en nitrate dans l'aquifère dépasse la norme eau potable $(50 \mathrm{mg} / \mathrm{l})$ sont amenées à décroître largement (de 17000 ha en 2003 à 4000 ha pour le scénario tendanciel et A1 ou 6000 ha pour B2 en 2015). Le scénario B2 qui induit un fort développement des cultures bioénergétiques (colza) conduit, alors qu'il a été construit avec davantage de contraintes environnementales, à un niveau de pollution supérieur au scénario tendanciel, suggérant un conflit potentiel entre les politiques et objectifs de réduction des émissions de gaz à effet de serre et de protection des ressources en eau.

Graphique 1. Évolution des surfaces (en ha) où les concentrations en nitrate dépassent les $50 \mathrm{mg} / \mathrm{l}$

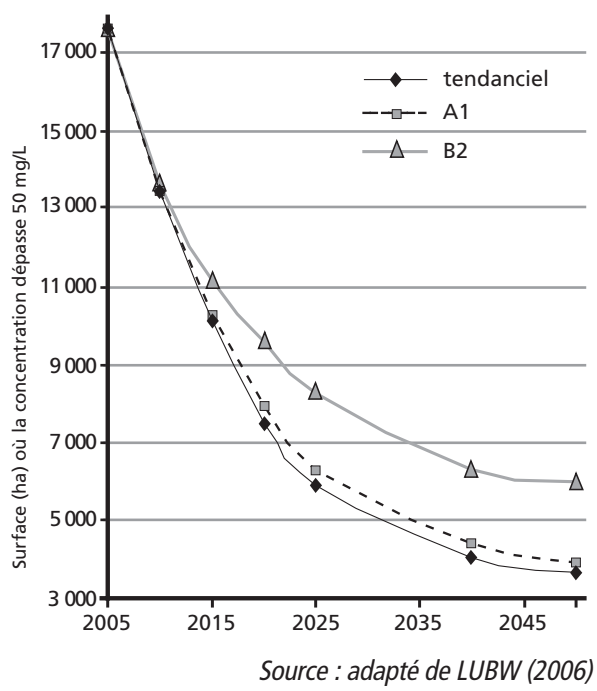

\section{Conclusion}

Les résultats de simulations présentés cidessus montrent que la baisse des concentrations en nitrates observée entre 1997 et 2003 devrait se poursuivre quelle que soit l'évolution future de l'environnement économique global. Si les tendances économiques anticipées se maintenaient jusqu'en 2027 (dernière échéance possible pour justifier une dérogation dans le cadre de la DCE), entre 6900 et 7700 hectares pourraient présenter des concentrations en nitrates supérieures à $50 \mathrm{mg} / \mathrm{l}$ à cette échéance (soit 2,5 à 3 fois moins qu'en 2003). De manière surprenante, le scénario B2 est celui qui conduit à la pire des situations du point de vue de la qualité de la 
nappe alors qu'il est conçu comme un scénario optimiste en matière de réduction d'émission de gaz à effets de serre. Ce constat s'explique en partie par le fort reliquat d'azote associé au colza dont les surfaces augmentent avec ce scénario. Il met en évidence le fait que des politiques environnementales peuvent être incompatibles entre elles, améliorant un problème environnemental tout en aggravant un autre. Les critères d'évaluation de politiques publiques dans le domaine de l'environnement devraient donc être élargis, pour rendre compte des effets directs et indirects des actions projetées sur les principaux milieux (eau, air sol), la biodiversité, l'émission de gaz à effets de serre, etc. Une telle évaluation environnementale élargie permettrait éventuellement d'identifier des synergies entre différentes politiques environnementales.

D'un point de vue méthodologique, le projet illustre comment une plateforme de modélisation intégrée peut être utilisée pour explorer de manière prospective l'évolution à long terme d'une ressource naturelle dont la dynamique dépend à la fois de facteurs et de processus de nature biophysiques et socio-économiques. Le recours aux modèles est alors clairement perçu comme une manière d'intégrer diverses hypothèses proposées par des experts, d'en évaluer leurs conséquences et de construire une vision partagée de futurs possibles. Il ne s'agit en aucun cas d'utiliser ces outils de simulation dans une logique de prédiction. Le développement de plusieurs variantes de scenarios d'évolution représente une façon de rendre compte des incertitudes associées à certains facteurs dont l'évolution est imprévisible à long terme, comme le marché des énergies fossiles ou les marchés des produits agricoles. Mais cette chaine de modèles pourrait aussi être utilisée pour simuler l'impact d'actions visant à accélérer l'amélioration de la qualité des eaux souterraines, permettant d'estimer le coût (via les modèles économiques) et l'effica- cité (via les modèles eaux souterraine) des différentes actions alternatives possibles afin de restaurer le bon état des masses d'eau aux horizons 2015, 2021 ou 2027.

Les modèles développés pourraient aussi être utilisés pour évaluer ex-ante l'efficacité ou l'impact de politiques visant à limiter les pollutions diffuses par les nitrates, bien qu'il faille considérer les problèmes d'information incomplète relatifs aux impacts environnementaux. Les instruments pouvant être évalués peuvent être classés dans quatre catégories :

- les mesures réglementaires telles que les doses maximales autorisées d'engrais par unité de terre ou par exploitation ;

- les marchés de quotas;

- la taxation des engrais ou bien des reliquats d'azote à la parcelle ;

- les mesures incitatives et / ou contractuelles telles que les mesures agro-environnementales (les Contrats d'Agriculture Durable en France par exemple). Un exemple d'application est donné dans Graveline et Rinaudo (2007) où l'impact des instruments de type taxe ont été simulés avec les modèles économiques présentés ici.

L'approche prospective présentée dans cet article présente cependant plusieurs limites qui réduisent la portée des résultats obtenus. La première limite est liée à la technique de modélisation économique adoptée. La typologie réalisée a ainsi été considérée comme statique alors qu'elle est en réalité dynamique. La réforme de la PAC va ainsi probablement conduire certains types à disparaître progressivement suivant des modèles démographiques (le type $\mathrm{C} 4$ par exemple) et d'autres à s'agrandir par rachat de terres. Une prise en compte de cette évolution de la typologie et de la structure des types qu'elle décrit dans la démarche de modélisation représente donc une piste de progrès. La seconde limite est liée aux incertitudes associées aux différents modèles et dont la propagation et les effets cumulatifs n’ont pas été étudiés. 


\section{RÉFÉRENCES BIBLIOGRAPHIQUES}

Alcamo J., Kreileman J., Bollen J.-C., van den Born J., Gerlagh R., Krol M.-S., Toet de Vries H. (1996). Baseline scenarios of global environmental change. Global Environmental Change 6(4): 261-303. Bartolini F., Bazzani G.-M., Gallerani V., Raggi M., Viaggi D. (2007). The impact of water and agriculture policy scenarios on irrigated farming systems in Italy: An analysis based on farm level multi-attribute linear programming models. Agricultural Systems 93(1-3): p.90-114.

Bouman B.-A.-M., Schipper R.-A., Nieuwenhuyse A., Hengsdijk H. and Jansen H.-G.-P. (1998). Quantifying economic and biophysical sustainability trade-offs in land use exploration at the regional level: a case study for the Northern Atlantic Zone of Costa Rica. Ecological Modelling 114(1): p.95-109.

Bouzit A.-M., Rieu T., Rio P. (1993). Modélisation du comportement des exploitations agricoles tenant compte du risque : application du MOTAD généralisé. Économie Rurale 220-221: p.69-73.

Casper M., Grimm-Strele J., Gudera T., Korte S., Lambrecht H., Schneider B., van Dijk P., Rinaudo J.-D. and Finck M. (2005) EU-Project MoNit: Decision support system to assess the impact of actions and changing frameworks on the nitrate load in the Upper Rhine Valley aquifer. Models and scenarios. Paper presented at the EWRA conference, Menton: September 7-10, 2005.

Chambre d'Agriculture région Alsace (2003) Typologie des exploitations alsaciennes. Schiltigheim : CRA. 45 p.

Deutscher Bauerverband (2003) Die Reform der Gemeinsamen AgrarPolitik - Deutscher Bauerverband - 16 p.

European Commission. 2002. Implementation of Council Directive 91/676/EEC concerning the protection of waters against pollution caused by nitrates from agricultural sources. Synthesis from year 2000 Member States reports. COM (2002) 407 fin. European Commission, Brussels.

Graveline N., Rinaudo J.-D, Segger V., Lambrecht H., Casper M., Grimm-Strele J., Van Dijck P., Koller R., Gudera T. and Elsass P. (2007) Integrating economic with groundwater modelling for developing long term nitrate concentration scenarios in a large aquifer. In Aquifer Systems Management: Darcy's legacy in a world of impending water shortage, Chery L and de Marsilly G (eds). AA. Balkema Publisher Chap. 36 - p. 483 -495 Graveline N., Rinaudo J.-D. (2007). Constructing scenarios of agricultural diffuse pollution using an integrated hydro-economic modelling approach. European Water Issue 17/18, 2007 disponible à http://ewra.net/ew/issue_17-18.htm

Guyomard H., Le Mouël C., Jez C., Forslund A., Fournel E. (2007). Prospective « Agriculture 2013 »- Résultats et enseignements principaux par thème. Paris, INRA. 55 p.

Hazell P.-B.-R. et Norton R.-D. (1986) Mathematical Programming for Economic Analysis in Agriculture. Macmillan New York. 400 p.

IFEN (2004) L'état des eaux souterraines en France : aspects quantitatifs et qualitatifs. Études et Travaux No. 43. 38 p.

Intergovernmental Panel on Climate Change (2000) Summary for policy makers Emissions scenarios. IPCC special report. $18 \mathrm{p}$.

Lacroix A., Beaudoin N., Makowski D. (2005). Agricultural water nonpoint pollution control under uncertainty and climate variability. Ecological Economics 53(1): p. 115-127.

Lacroix A., Bel F., Mollard A., Sauboua E. (2007). Interest of site-specific pollution control policies: the case of nitrate pol- 
lution from agriculture. Int. J. Agricultural Resources, Governance and Ecology 6(1): p. 45-59.

Lacroix A., Mollard A. (2002). Bilan scientifique d'une recherche interdisciplinaire entre agronomes, hydrologues et économistes : le point de vue des économistes. Nature Sciences Sociétés 10(1): p. 7-14.

LUBW (2006) Modélisation de la pollution des eaux souterraines par les nitrates dans la vallée du Rhin supérieur. Rapport final. Accessible à : www.lubw.badenwuerttemberg.de $>$ Publikationen $>$ Wasser.

Marleix A. (2004). Rapport sur les biocarburants présenté par la commission des finances, de l'économie générale et du plan. Assemblée nationale ${ }^{\circ} 1622$.

MAPAAR, Ministère de l'agriculture de l'alimentation de la pêche et des affaires rurales (2004). La nouvelle politique agricole commune (PAC) : les modalités d'application nationale 2005-2006-2007. Paris MAPAAR.

Nidumolu U.-B., van Keulen H., Lubbers M., Mapfumo A. (2007). Combining interactive multiple goal linear programming with an inter-stakeholder communication matrix to generate land use options. Environmental Modelling \& Software 22(1): p. 73-83.

Région Alsace (2000) Inventaire de la qualité des eaux souterraines dans la vallée du Rhin Supérieur 1997 - Strasbourg

Rehman T., Romero C. (1993). The appli- cation of the MCDM paradigm to the management of agricultural systems: Some basic considerations. Agricultural Systems 41(3): p.239-255.

Rinaudo J.-D., Arnal C., Blanchin R., Elsass P., Mailhac A., Loubier S. (2005) Assessing the cost of groundwater pollution: the case of diffuse agricultural pollution in the Upper Rhine valley aquifer, Water Science and technology, 52 (9) - p. 153-162

Rinaudo J.-D., Graveline N., Elsass P. (à paraître). Combining hydrogeological and economic approaches and models for designing groundwater protection programmes of measures in the Upper Rhine valley aquifer. In M. Pulido, I. Heinz, J. Lund, J. Andreu (Eds), Economics in water management models.

Rozakis S., Sourie J.-C., Vanderpooten D. (2001). Integrated micro-economic modelling and multi-criteria methodology to support public decision-making: the case of liquid bio-fuels in France. Biomass and Bioenergy 20(5): p. 385398.

Ruben R., Moll H., Kuyvenhoven A. (1998). Integrating agricultural research and policy analysis: analytical framework and policy applications for bio-economic modelling. Agricultural Systems 58(3): p. 331-349.

Zander P., Kächele H. (1999) Modelling multiple objectives of land use for sustainable development. Agricultural Systems 59 (3): p. 311-325. 